$P=s . \exp (E / k T):$ common ground in thermal physics

\section{G.F.J. Garlick}

Analysis of Thermally Stimulated Processes. By R. Chen and Y. Kirsh. Pp.361. ISBN 0-08-022930-1. (Pergamon: 1981.) $\$ 60, £ 25$.

WHAT might there be in common among such apparently diverse experimental operations as the thermal stimulation of luminescence or current on the one hand, and thermogravimetry or differential thermal analysis on the other? Add to these other processes measured in, for example, thermal annealing, evolved gas analysis, thermoremanent magnetism, thermal desorption, dilatometry and thermally induced creep, and there is the subject matter of the present text.

In their preface, the authors themselves make the comment that the book could look like a "marriage of convenience". However, there is more connection between the effects than that they each involve a change in some measurable physical parameter when a material sample is warmed up. The more subtle factor common to them all is the theoretical expression for the probability of the effect, which is the familiar Arrhenius exponential function $P=s \cdot \exp (E / k T)$, where $s$ is often known as the "frequency constant", $E$ is the energy needed to effect the change in parameter, $k$ is Boltzmann's constant and $T$ the absolute temperature. The major problems of developing theory to correlate with any of them are not about this function but about the kinetics which determine what happens later. For instance, will an escaping electron recombine with its original location to give, for example, luminescence, or will it be retrapped?

A large part of the book is taken up with these kinetic variations and the reader interested in thermogravimetry or differential thermal analysis may find that his interests are somewhat overwhelmed by extensive treatments of thermally stimulated luminescence and conductivity. The volume will certainly not replace the many treatises on thermogravimetry or differential thermal analysis and other specific techniques to which the users of the associated, sophisticated equipment now turn. However, there is always the possibility that by reading of the existence of a wider range of thermally stimulated effects ideas may be transferred between the disciplines. In this sense the text will be very useful.

With the major concern of the authors being thermally stimulated electronic processes in insulators and semiconductors, there is a fairly exhaustive coverage of the theory and practice of studying "deep" levels in such solids. There are, however, some omissions and some inaccuracies. On a historical note, more credit should have been given to
Urbach for his quantitative assessment of thermoluminescence in 1930 which preceded that of Randall and Wilkins by many years. On the matter of retrapping the authors do not, in drawing examples from the literature, bring out the stillpuzzling case of zinc sulphide phosphors, where luminescence and conductivity can be co-explored to determine detailed mechanisms. In 1948 Wilkins and I (Nature 161, 565-566) showed that retrapping of thermally released electrons was very small even when empty traps outnumbered empty recombination centres by more than 1000:1. In another instance, when reviewing photon-induced transfer of carriers between trapping states, the enormous effect found in infrared-stimulable zinc sulphides by myself and Mason ( $J$. Electrochem. Soc. 96, 90-113;1949) is not referred to. These are important examples because they indicate ways in which retrapping can be examined in an important group of high band gap solids.

In spite of the problems of using thermally stimulated processes to obtain accurate values of trap energies and crosssections, the recent development of DLTS (deep level transient spectroscopy) as a steady state method of thermal stimulation would appear to offer a breakthrough for determination of these parameters in device semiconductors such as silicon and gallium arsenide. It is a pity that some of the elegant examples of trap distributions obtained by this method were not included in the text.

There is no doubt that this book will be attractive to particular groups of readers who, like myself, have specific interests in carrier trapping and related processes in insulators and semiconductors. It is well referenced and has a critical approach to the use and interpretation of thermal stimulation methods. Whether its attempt to coordinate analysis of the many different thermally stimulated effects will appeal to a wider readership is less sure.

G.F.J. Garlick is a consultant specializing in the fields of luminescence and electronic processes in semiconductors.

\title{
Developments in theorists' views of ecology
}

\section{Richard Law}

Theoretical Ecology: Principles and Applications, 2nd Edn. Edited by Robert M. May. Pp.489. ISBN hbk 0-632-00768-0; ISBN pbk 0-632-00762-1. (Blackwell Scientific/Sinauer Associates: 1981.) Hbk $£ 20$, $\$ 42.75$; pbk $£ 10.50, \$ 23.95$.

THEORETICAL ecology is a fast growing subject due, in large measure, to the stimulus of R.M. May and his associates; it is pleasing, therefore, that they should take the time to produce a new edition of their standard work (first published in 1976).

While retaining the same overall theme of developing a theoretical perspective from which to view the living world, the new edition contains some substantial changes. Several chapters have been modified in the light of recent work - most notably that on patterns in multi-species communities, which now considers intriguing questions such as how many trophic levels should exist and how theories about community stability and complexity can be tested. Several other chapters have been rewritten by new authors; in particular, that on plant-herbivore systems has been improved by taking greater account of herbivory in the real world. However other chapters that would have benefited from revision remain unchanged. For example, "Island Biogeography and the Design of Nature Reserves" hardly does justice to the promise of its title, design principles for nature reserves being relegated to a few paragraphs at the end. No mention is made of recent work in this area and of the debate that has come to surround the whole subject.

There are two welcome additions to the book. The first is a survey of the new kind of population dynamics that is emerging from studies of plants, in which individuals, rather than growing to a fixed size, may continue to grow indefinitely by the production of modular units of construction. The second, dealing with the economics of biological resources, follows an elegant path from ecological theory to practical questions of optimal harvesting and the regulation of fisheries.

The book does not attempt to give a comprehensive coverage of theoretical ecology - for example, the reader will search in vain for an adequate account of the dynamics of populations with age distributions or of the effects of genetic variability in ecological parameters. But any shortcomings are greatly outweighed by its merits; it is a lucid and up-to-date introduction to the contributions of R.M. May and his associates to theoretical ecology, and it deserves to be widely read by all those with an interest in the subject.

Richard Law is a Lecturer in the Botany Depart. ment at Sheffield University. 